\title{
Investigation of Various Techniques of Spectrum Sensing for Power Detection in Cognitive Radio
}

\author{
M. Monisha ${ }^{1^{*}}$, C. Sharanya ${ }^{2}$, M. Meena ${ }^{3}$, V. Rajendran ${ }^{4}$ \\ ${ }^{1}$ Research Scholar, Department of ECE, VELS Institute of Science, Technology and Advanced Studies, Chennai. \\ ${ }^{2}$ Research Scholar, Department of ECE, VELS Institute of Science, Technology and Advanced Studies, Chennai. \\ ${ }^{3}$ Research Scholar, Department of ECE, VELS Institute of Science, Technology and Advanced Studies, Chennai. \\ ${ }^{4}$ Prof \& Head, Department of ECE, VELS Institute of Science, Technology and Advanced Studies, Chennai.
}

\begin{abstract}
The most competitiveness among the remote clients in India, transfer speed turns into the noticeable factor. Every one of the clients search for financially savvy and frightfully proficient frameworks to utilize the data transfer capacity viably for the essential and the auxiliary client. In this association, the current research on various MAC layers plainly uncovers that MC-CDMA procedure is giving a promising information throughput and better ghastly productivity. In the current present day advancement the medium range correspondence frameworks like dispatch administrations, roadway administrations, inn segment administrations is winding up exceptionally prevalent and every one of these applications needs an organized range detecting, range openings, range sharing, range administration, and range planning. Every one of these variables is kept up by intellectual radio approach. In this paper we examine about different procedures of range detecting in intellectual radios and its energy phantom thickness is assessed.
\end{abstract}

Keywords: Cognitive radio, radio spectrum, spectrum sensing, spectrum mobility, spectrum management, spectrum sharing, power spectral density.

\section{Introduction to Cognitive Radio}

Joseph Mitola III [3] was the person who proposed the idea of psychological radio in a class in 1998 and distributed in an article in 1999. Intellectual radio is a wise based framework which can distinguish the best remote correspondence directs being used, and move into those empty channels by staying away from the obstruction of the clients in its locale.

In regular method every client is allotted a permit recurrence band to work. The allotted range stays unused or not utilized adequately. To beat range deficiency and underutilization, psychological radio systems is presented which effectively use the radio range and makes correspondence more dependable for clients unexceptionally. The one of a kind normal for subjective radio framework is to recognize the electromagnetic condition and capacity to find out about it and changing its working parameters powerfully. This strategy of using the unused range band is known as unique range get to (DSA).

Cognitive radio architecture is shown in "Fig. 1 "

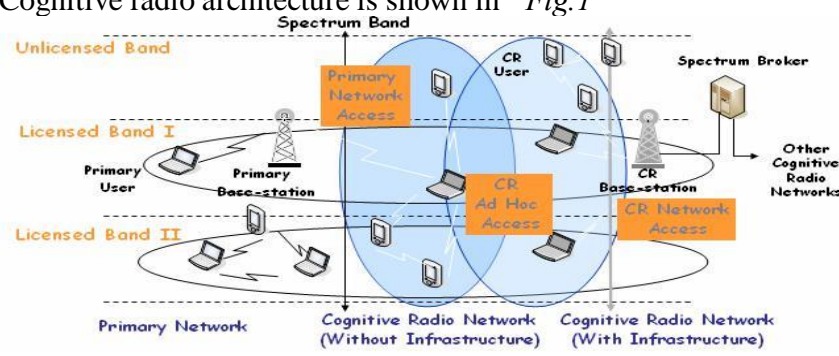

Fig. 1: Cognitive radio architecture
"Fig. 2", demonstrates the range opening identification and powerful use of the authorized band without essential client for the optional client.

Range openings are a band of frequencies which are doled out to the essential clients which is the authorized proprietor of the band, at a specific time and at particular geographic areas however the band is underutilized by the client.

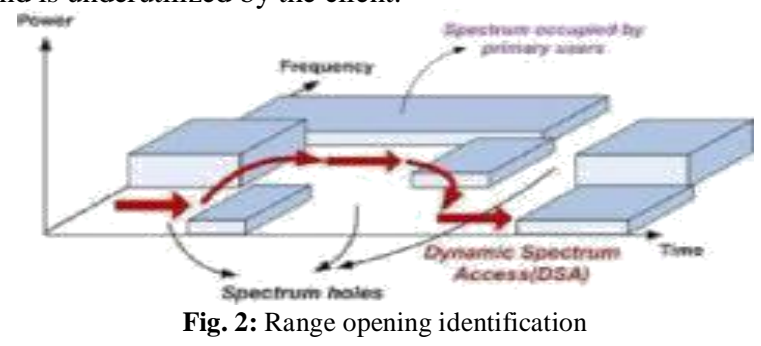

\section{Features of Cognitive Radio}

There are two fundamental highlights [5]

- Cognitive ability: The intellectual radio sense the data from the radio condition to locate the unused radio range at that area.

- Re-configurability: Ability of the radio to change its capacities and it is modified powerfully as per radio condition. "Fig. 2", shows the importance of spectrum hole detection and how the secondary user efficiently utilize the licensed band in absence of primary user.

Spectrum holes are a band of frequencies which are assigned to the primary users which is the licensed owner of the band, at a particular time and at a specific geographic locations but the band 
is underutilized by the user. Spectrum hole concept can be further generalized as transmission opportunity in a radio spectrum space.

\section{Functions of Cognitive Radio}

The 4 noteworthy elements of subjective radio cycle are appeared in "Fig. 3"

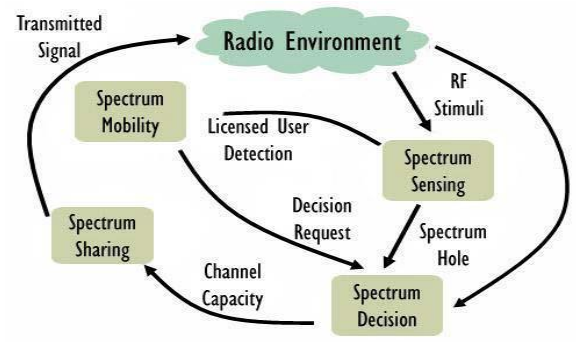

Fig. 3: Subjective radio cycle

It identifies the nearness of the essential clients in the band. In the wake of detecting the essential client's range it imparts its outcomes to the next intellectual radio. The primary part of range detecting is to know the status of the objective recurrence band by occasionally detecting it. The handset of subjective radio screens the range gap and an approach to get to it without meddling.

\section{Spectrum Administration}

It is the way toward guiding the utilization of radio frequencies to advance proficient and powerful utilize. Expanding interest for administrations has required changes in standpoint of range administration. The accessible range gap in the channel is instantly chosen by the psychological radio. It comprises of range detecting, range examining and range choice. Range examination influences conceivable to get fitting range to band according to client necessity. Range choice alludes to choosing the information rate, transmission mode and data transfer capacity as per client necessities.

\section{Spectrum Sharing}

Range partaking in psychological radio enables the client to share the range band of the authorized client. Notwithstanding, the psychological radio clients need to limit their transmitting energy to decrease the obstruction caused to the authorized band clients which is to be kept beneath a specific edge level.[3]

\section{Spectrum Versatility}

Range versatility in the sense, the subjective radio clients are permitted to change their working recurrence. This can likewise be called as handoff. It works in the accessible recurrence band of the range and keeps up continuous correspondence.

\section{Spectrum Sensing Methods}

\section{Matched filter}

The coordinated channel is the standout amongst other locator for Gaussian commotion which has high flag to-clamor proportion. In this strategy, less recognition time is required for higher preparing pick up [5]. This strategy can be viably utilized just when the data of authorized clients is known to intellectual clients likewise called the optional clients.

The piece outline of coordinated channel is appeared in figure 4 .

The contribution to coordinated channel is the flag got by the CR,

$\mathrm{a}(\mathrm{t})=\mathrm{s}(\mathrm{t})+\mathrm{n}(\mathrm{t})$ $\mathrm{r}(\mathrm{t})$ convolves with $\mathrm{h}(\mathrm{t})$ and this is performed by the coordinated channel.

Where,

$h(t)=s(T-t+\tau)$

The yield of coordinated channel is contrasted at long last and a limit esteem $\lambda$ to choose whether the essential client is available or not.

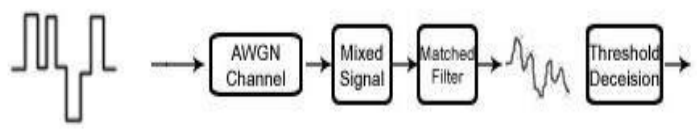

Fig. 4: Square diagram of matched filter

The coordinated channel strategy is as per the following. Stage 1: Generate neighborhood bearer utilizing nearby oscillator since the earlier information of essential client waveform is required or the coordinated channel.

Stage 2: Gauge the cross-relationship succession of an arbitrary procedure utilizing xcorr. Autocorrelation is an uncommon case.

Stage 3: think about the outcomes at low and high SNR and afterward edge $\lambda$ is set to be \pm 35 .

Stage 4: look at the yield of the integrator, Y with a limit esteem $\lambda$ to choose whether essential client is available or not.

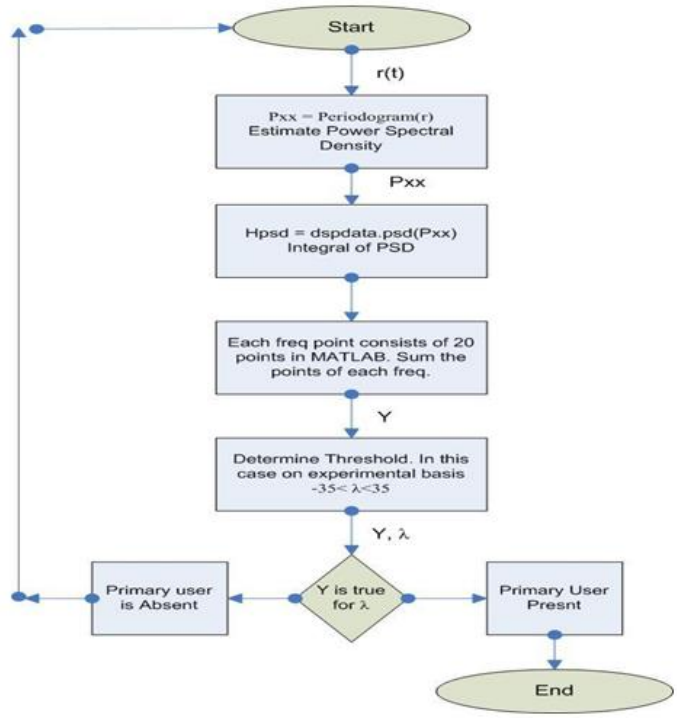

Fig. 5: Stream graph for implementation of matched filter

The connection of got motion with flag created at psychological radio under great SNR conditions is appeared in figure 6 .

- A earlier learning of each authorized client flag is required.

- A collector which is committed for a wide range of flag is required, so the usage multifaceted nature is illogically more prominent in detecting unit. [6]

- Large number of collector calculations should have been executed for location because of huge power utilization. [6]

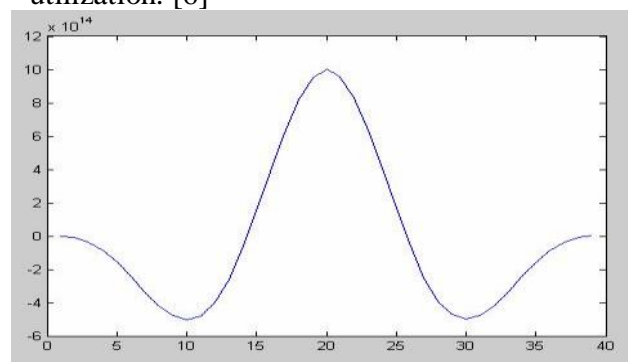

Fig. 6: Age of flag at subjective radio under great SNR conditions 
Vitality location is the most well-known method for range detecting likewise called non cognizant discovery technique which has low computational and usage complexities [1], which is utilized to distinguish the nearness of the essential client flag.

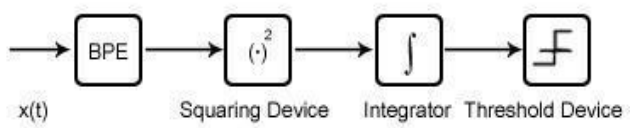

Fig. 7: Block diagram of energy detector

Let the got flag $\mathrm{r}(\mathrm{t})$ which we need to go from vitality finder.

The Energy Detector system is as per the following.

Stage 1: Estimate the Power Spectral Density (PSD) by utilizing periodogram work in MATLAB.

Pxx $=$ Periodogram $(r)$

Stage 2: Compute the normal power in the flag over the recurrence band from the essential of the PSD over that given recurrence band.

$$
\text { Hpsd=Dspdata.psd (Pxx) }
$$

Stage 3: Obtain the outcome in MATLAB by summing up all the 20 focuses contained in one recurrence part

Stage 4: Compare the outcomes at low and high SNR and afterward set the limit esteem $\lambda$ to be 5000 .

Stage 5: Compare the yield of the integrator, $\mathrm{Y}$ with a limit esteem $\lambda$ to choose whether essential client is available or not.

It's obvious from the figure 9 that there is crest at precisely 200

Hz. So vitality identifier contrasts this pinnacle and edge esteem and for this situation it's observed to be more noteworthy than the limit. Subsequently, it is gathered from the vitality identifier that the essential client is available at $200 \mathrm{~Hz}$.

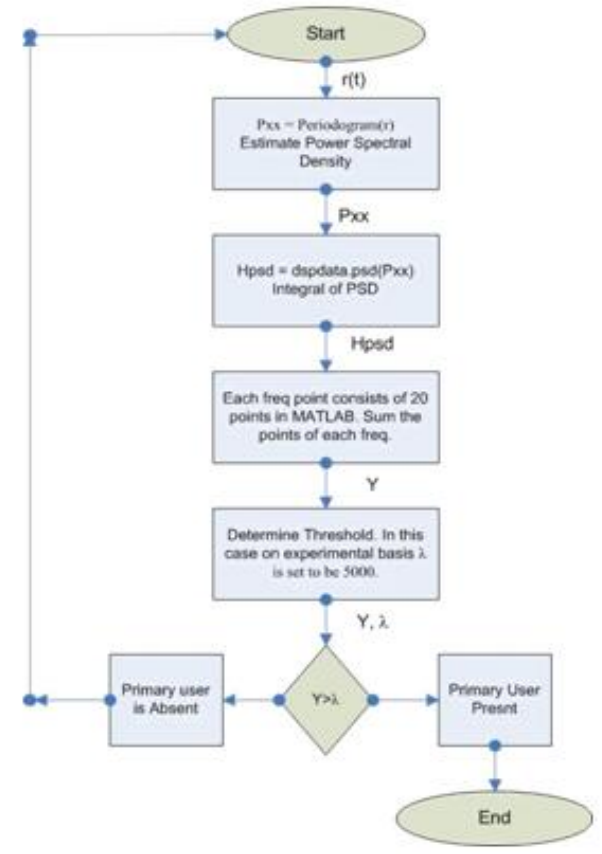

Fig. 8: Stream graph for implementation of energy detector

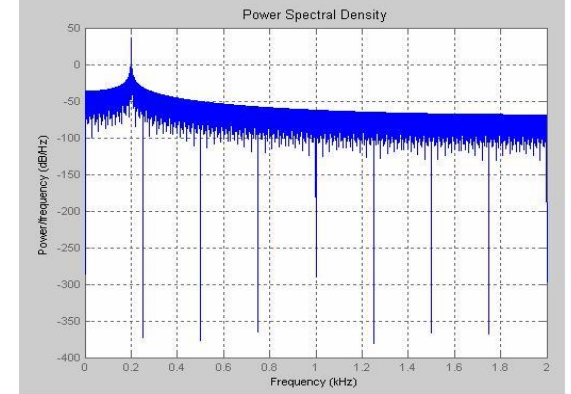

Fig. 9: Power spectral density output of energy detector using BPSK

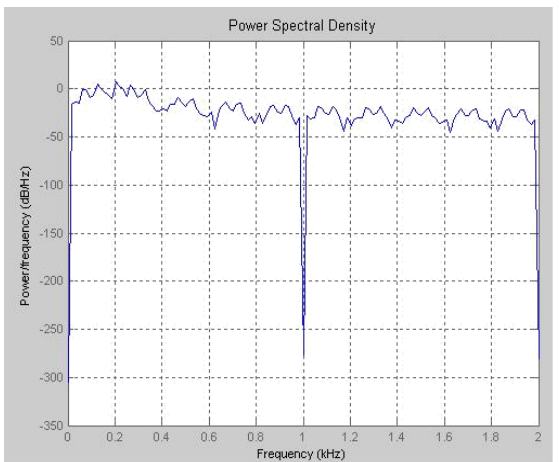

Fig. 10: Power spectral density output of energy detector using QPSK

\section{Drawbacks}

- Unable to separate between the impedance and essential client clamor

- Poor execution under low Signal-to-clamor proportion.

\section{Cyclostationary feature detection}

Cyclostationary discovery depends on the intermittent repetition which is presented in the flag by balance and testing process. The periodicity of essential client flag transmission is distinguished by accomplishing the cyclostationarity highlights of the received signals. [6][1][2].

Think about $r$ (t) to be the gotten flag to be passed from cyclostationary highlight identifier. The method of the Cyclostationary Feature Detection is as per the following.

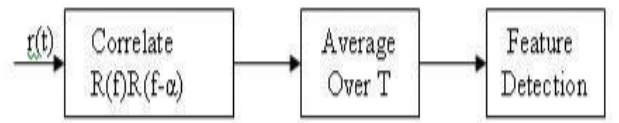

Fig. 11: Block Diagram of cyclostationary feature detector

Stage 1: Compute the Fourier of the got motion by utilizing 'fft' work.

$\mathrm{A}=\mathrm{fft}(\mathrm{a})$

Stage 2: Multiply $r$ with complex exponential.

Increase with complex exponential in time space is comparable to recurrence move in recurrence area.

$\mathrm{XT}=\mathrm{r} . * \exp (\mathrm{j} * 2 * \mathrm{pi} * \mathrm{shfT})$;

Stage 3: Compute Correlation of XT with A

$\mathrm{XY}=\mathrm{x} \operatorname{corr}(\mathrm{XT}, \mathrm{A})$

Perform Average after some time T

$\mathrm{pt}=\mathrm{fft}(\mathrm{XY}) \cdot{ }^{*} \operatorname{conj}(\mathrm{fft}(\mathrm{XY}))$ 
Stage 4: Compare the outcomes at low and high SNR and set the limit an incentive to be $1<\lambda<5$.

Stage 5: Compare the yield of the integrator, pt with a limit esteem $\lambda$ to choose whether essential client is available or not.

In this manner if the essential client is available then we can discover highlights of the essential flag like working recurrence and adjustment system.

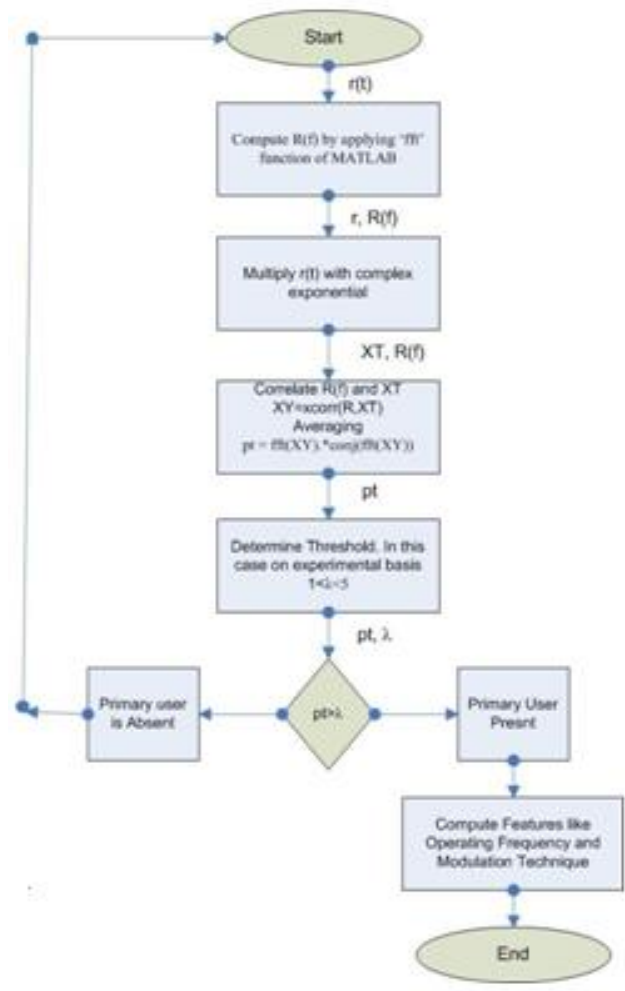

Fig. 12: Flow chart for the cyclostationary feature detection implementation

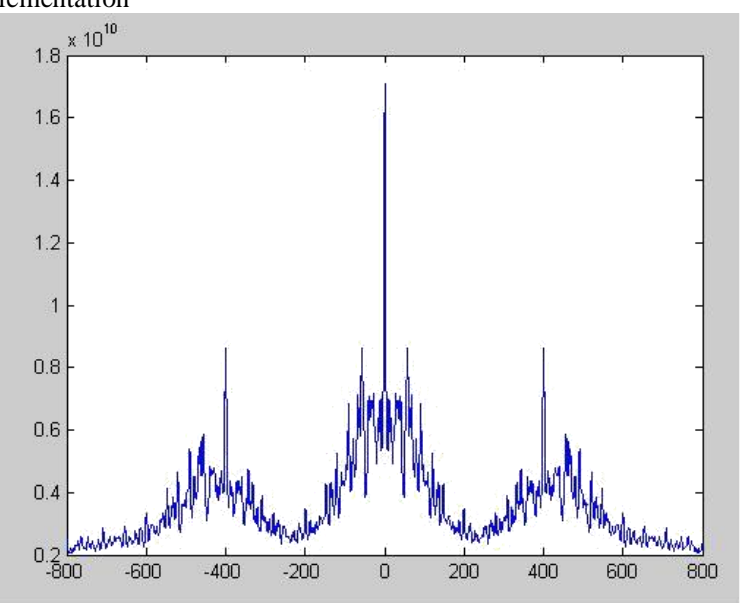

Fig. 13: Cyclostationary feature detector output at SNR 30dB for BPSK

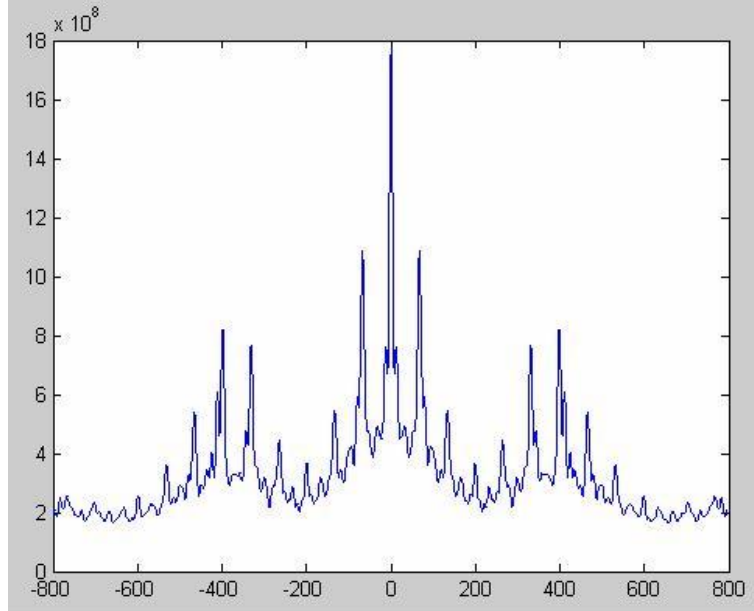

Fig. 14: Cyclostationary feature detector output at SNR 30dB for QPSK when essential client is available at $200 \mathrm{~Hz}$

Cyclostationary recognition has a hindrance of high computational complexities and longer time for perception.

\section{Radio identification technique}

This method is used in the context of European Ubiquitous Terminal (TRUST) project (Farnham et al., 2000). A complete knowledge about the spectrum characteristics is identified by the transmission technologies used by primary users. Earlier information about the range qualities like transmission go, balance method, go removed from the got flag, and psychological radio chooses the best appropriate transmission parameter. [9]

\section{Waveform based sensing}

This procedure is utilized as a part of picture preparing for edge recognition applications. This approach is primarily connected in range detecting of CRs where wavelets are utilized for identifying the limit between the range openings and possessed groups. Henceforth the empty range groups are anything but difficult to recognize. In view of this data $\mathrm{CR}$ can likewise distinguish the range openings.

a) Sensing Time: During correspondence, intellectual radio ceaselessly sense the radio condition for range openings. In this manner it is required for the detecting time to be as least as would be prudent. Coordinated Filtering is a decent strategy for range detecting in psychological radio systems without having any earlier information about essential clients waveform. Be that as it may, in the vast majority of the cases, the earlier learning about essential client's waveform is obscure, which makes it troublesome for the utilization of range detecting. Henceforth it requires less detecting time to accomplish high preparing pick up. Contrasting Energy Detector and Matched Filtering, Energy Detector requires a more drawn out detecting time to accomplish great outcomes as appeared in Table 1.

Cyclostationary Feature Detection is likewise a non sound procedure which makes it better than Matched Filtering. The calculation is exceptionally mind boggling and it requires longer perception investment for sensing.[6]

Table 1: Sensing time for Transmitter Detection Techniques

\begin{tabular}{|l|l|l|l|}
\hline $\begin{array}{l}\text { Sort of essential } \\
\text { signal }\end{array}$ & $\begin{array}{l}\text { Energy } \\
\text { detection }\end{array}$ & $\begin{array}{l}\text { Matched } \\
\text { filter }\end{array}$ & $\begin{array}{l}\text { Cyclostationary based } \\
\text { sensing }\end{array}$ \\
\hline BPSK & $1.20 \mathrm{sec}$ & $0.17 \mathrm{sec}$ & $9.50 \mathrm{sec}$ \\
\hline QPSK & $1.30 \mathrm{sec}$ & $0.20 \mathrm{sec}$ & $11.21 \mathrm{sec}$ \\
\hline
\end{tabular}


Consequently from the trial brings about Table 1 demonstrates that coordinated channel requires slightest among the all detecting procedures and cyclostationary takes most.

b) Simple Implementation: Low cost and straightforward usage, which is required for range detecting in intellectual radio systems. [8]Coordinated Filter isn't anything but difficult to actualize in light of the fact that it requires producing bearer at beneficiary, which expands the cost of subjective radio. Cyclostationary Feature Detection is additionally extremely complex procedure which takes high cost and high computational complexity.[4]

Table 2: Comparison of Transmitter Detection Techniques

\begin{tabular}{|l|l|l|l|l|}
\hline $\begin{array}{l}\text { SR } \\
\text { No. }\end{array}$ & Type & $\begin{array}{l}\text { Energy } \\
\text { Detection }\end{array}$ & $\begin{array}{l}\text { Matched } \\
\text { filter }\end{array}$ & $\begin{array}{l}\text { Cyclostationary } \\
\text { based sensing }\end{array}$ \\
\hline 1. & Sensing Time & More & Less & Most \\
\hline 2. & Easy to Implement & Yes & No & No \\
\hline 3. & Performance under noise & Poor & Bad & Good \\
\hline 4. & Prior Knowledge Required & No & Yes & No \\
\hline
\end{tabular}

\section{Applications of Cognitive Radio}

The uses of subjective radio are classified into three sorts.

- Short go correspondence application incorporates telecom companies, home machines and some indoor applications like home computerization, manufacturing plant mechanization, telemedicine, and so on.

- Medium extend correspondence application incorporates coordinations, activity observing, vehicle following, stock following, catastrophe alleviation tasks and some military applications.

- Long go correspondence application incorporates portable systems, Industrial remote robotization, military and open security applications, and so on [2].

\section{Conclusion}

In this paper we have proposed various techniques of spectrum sensing in cognitive radios and its power spectral density is estimated. The sensing times are being calculated for BPSK and QPSK signals using different spectrum sensing techniques and its comparison is tabulated. The different application of cognitive radio technology is studied. In future, the design and development of Software Defined Radio algorithm for a few medium range communication applications is to be implemented.

\section{References}

[1] Ghosh G, Das P \& Chatterjee S, "Simulation and analysis of cognitive radio system using Matlab", International Journal of Next-Generation Networks, Vol.6, No.2,(2014).

[2] Hemalatha M, Prithviraj V, Jayalalitha S, Thenmozhi K, Bharadwaj D \& Girish GK, "A survey report on spectrum sensing techniques in cognitive radio", Journal of Theoretical and Applied Information Technology, Vol. 37, No.1, (2012), pp.32-38.

[3] Mitola J, "Cognitive radio: an integrated agent architecture for software defined radio", Ph.D. Dissertation, KTH Royal Institute of Technology, Stockholm, Sweden, (2000).

[4] Surendran R, Muthumeenakshi K \& Radha S, "A distributed algorithm for dynamic spectrum access using primary-prioritised markov model", International Conference on Process Automation, Control and Computing (PACC), (2011), pp.1-5.

[5] Saloni PB, "Spectrum Sensing in Cognitive Radio by Statistical Matched Wavelet Method and Matched Filter", International Journal of Electronics \& Communication Technology, Vol.7, No.1, pp.33-39.
[6] Yucek T \& Arslan H, "A survey of spectrum sensing algorithms for cognitive radio applications", IEEE communications surveys \& tutorials, Vol.11, No.1,(2009), pp.116-130.

[7] Akyildiz IF, Lee WY, Vuran MC \& Mohanty S, "NeXt generation/ dynamic spectrum access/cognitive radio wireless networks: A survey", Computer networks, Vol.50, No.13,(2006), pp.2127-2159.

[8] Z Yesembayeva (2018). Features of the legal status of judges: Kazakhstan experience and foreign realities Opción, Año 33. 447474

[9] Akhpanov, S. Sabitov, R. Shaykhadenov (2018). Criminal pre-trial proceedings in the Republic of Kazakhstan: Trend of the institutional transformations. Opción, Año 33. 107-125. 